\title{
Thermodynamics and Hawking radiation of five-dimensional rotating charged Gödel black holes
}

\author{
Shuang-Qing $\mathrm{Wu}^{*}$ and Jun-Jin Peng ${ }^{\dagger}$ \\ College of Physical Science and Technology, HuaZhong Normal University, \\ Wuhan, Hubei 430079, People's Republic of China
}

\begin{abstract}
We study the thermodynamics of Gödel-type rotating charged black holes in five-dimensional minimal supergravity. These black holes exhibit some peculiar features such as the presence of closed time-like curves and the absence of globally spatial-like Cauchy surface. We explicitly compute their energies, angular momenta, and electric charges that are consistent with the first law of thermodynamics. Besides, We extend the covariant anomaly cancellation method, as well as the approach of the effective action, to derive their Hawking fluxes. Both the methods of the anomaly cancellation and effective action give the same Hawking fluxes as those from Planck distribution for blackbody radiation in the background of the charged rotating Gödel black holes. Our results further support that Hawking radiation is a quantum phenomenon arising at the event horizon.
\end{abstract}

PACS numbers: 04.70.Dy, 04.62.+v

\section{INTRODUCTION}

Gödel universe is a model that describes the universe with a global rotation. In four dimensions, the Gödel universe is an exact solution of Einstein field equation with a negative cosmological constant and homogeneous pressure less matter, found by Gödel in 1949 [1]. Unlike the usual solutions in general relativity, this solution possesses some peculiar features such as the allowance of closed time-like curves and the absence of globally spatial-like Cauchy surface. It is of great importance for the conceptual development of general relativity. In recent years, much of interest has been focused on various generalizations of the four dimensional Gödel universe, particularly in the context of the five dimensional minimal supergravity theory [2 8$]$. Just as in Gödel's original four dimensional solution, all the higher dimensional generalized solutions present closed time-like curves for all times. Furthermore, they can be easily uplifted to $\mathrm{M}$ theory. A remarkable observation also showed that the maximally supersymmetric analogues of the Gödel universe in [2] are T-dual to pp-waves [3].

Among all the Gödel-type generalizations in five dimensional minimal supergravity, one solution describing a stationary Kerr black hole embedded in the rotating Gödel universe was recently found by Gimon and Hashimoto [4]. This solution is not required to preserve any supersymmetry, compared with the supersymmetric one in [2]. Its various properties have been intensively investigated in 9-14, 21]. Particularly in [9], Barnich and Compère proposed an effective method to calculate conserved charges in the Gödel-type background. They obtained the Kerr Gödel black hole's conserved charges that fulfill the first law of thermodynamics. The charged generalization of the Kerr Gödel black hole has been found by one of the authors [8]. Such a solution is an analytic solution in five dimensional Einstein field equation coupled with Maxwell and Chern-Simons terms in Gödel background. We shall refer to it as Einstein-Maxwell-Chern-Simons-Gödel (EMCS-Gödel) black hole. After getting this black hole solution, it is very necessary to study its thermodynamical properties. In this paper, we explicitly compute the mass, angular momenta and electric charge of the EMCS-Gödel black hole along the lines of [9]. These conserved charges satisfy the differential first law and the generalized integral Smarr formula of black hole thermodynamics. However, unlike work [9], to close the integral Smarr formula, the Gödel parameter is seen as a thermodynamical variable [8]. For the extremal EMCS-Gödel black holes, their microscopic entropies can be derived through Kerr/CFT correspondence [14].

In the above, we have mentioned that the EMCS-Gödel black hole exhibits thermodynamical characters. Thus there must exist Hawking radiation at its event horizon. This quantum phenomenon is actually very universal and can be found in any geometry background with event horizons. It is regarded as a clue for seeking the theory of quantum gravity. Although Hawking radiation has not yet been observed on laboratory, it has been verified by several different approaches since Hawking discovered this effect more than thirty years ago. Recently, Wilczek and his collaborators proposed a new derivation of Hawking radiation from four dimensional black holes via gravitational and gauge anomalies 16 18]. In their works, Hawking radiation is treated as a compensating flux to cancel gravitational and gauge anomalies at the horizon, which arise since the effective field theory becomes two dimensional and chiral

\footnotetext{
* Electronic address: sqwu@phy.ccnu.edu.cn

$\dagger$ Electronic address: pengjjph@163.com
} 
after performing a procedure of dimensional reduction near the horizon of a black hole. This anomaly cancellation method supports that Hawking radiation is a common property of the horizon. It is very universal and has been successfully applied to black objects in various dimensions [19 30. Noticing that the anomalous energy momentum tensors and currents encompass two types of forms in the two dimensional chiral effective theory, apart from the consistent form in [16 18], the other type is the covariant one. In [27], it was argued that Hawking fluxes of energy momentum tensors and gauge currents can be obtained by cancelling the covariant gravitational anomaly and gauge anomaly at the horizon. Such an argument makes the original anomaly cancellation method [16 18$]$ more economical and conceptually cleaner. Based on development in [27], several extensions can be found in [28 30]. Especially in 29], Hawking radiation of black strings in four and higher dimensions has been studied via covariant anomalies.

A notable feature of the anomaly cancellation method is that the boundary conditions at the event horizon play an important role in determining the Hawking fluxes. Indeed, in [31], by only imposing the boundary condition that the covariant energy momentum tensor and the covariant gauge current vanish at the horizon, the chiral effective action, which describes the two dimensional chiral theory near the horizon, has been used to compute the Hawking fluxes of charged spherically symmetric black holes. This effective action method is very universal and holds true for other black holes 21, 25, 33]. In addition to the chiral effective action, the normal effective action that induces anomaly free energy momentum tensors and gauge currents has also reproduced the Hawking fluxes of the Reissner-Nordström black hole [18]. A lot of works on applying the effective action to study Hawking effect can be found in [34 37].

In this paper, we investigate the thermodynamics of the EMCS-Gödel black hole and then generalize the covariant anomaly cancellation method, as well as the effective action approach, to study its Hawking radiation. Both the methods present the same Hawking fluxes. Our results support that Hawking radiation is a universal quantum phenomenon arising at the event horizon. The remainder of this paper goes as follows. In section II, we calculate the mass, the angular momenta and the electric charge of the EMCS-Gödel black hole, which satisfy the first law of thermodynamics. In section III, we compute the Hawking fluxes by treating them as compensating fluxes to cancel the covariant gravitational and gauge anomalies near the horizon. In section [V] we reproduce the Hawking fluxes of the EMCS-Gödel black hole via the approach of the effective action, including the normal effective action in subsection IVA and the chiral effective action in subsection IVB. The last section is our conclusions.

\section{THERMODYNAMICS OF THE EMCS-GÖDEL BLACK HOLE}

In this section, we study the thermodynamics of the EMCS-Gödel black hole [8]. Although the main results were presented in [8], here we give the explicit calculations by adopting the gauge field whose electric-static potential vanishes at infinity. Our starting point is the EMCS-Gödel black hole, which is a non-extremal charged rotating Gödel-type black hole solution in five-dimensional ungauged minimal supergravity. The relevant Einstein-Maxwell Lagrangian with Chern-Simons term reads

$$
L=\frac{\sqrt{-g}}{16 \pi}\left(R-F_{\mu \nu} F^{\mu \nu}\right)-\frac{1}{24 \pi \sqrt{3}} \epsilon^{\lambda \rho \sigma \mu \nu} A_{\lambda} F_{\rho \sigma} F_{\mu \nu},
$$

where $\epsilon^{\lambda \rho \sigma \mu \nu}$ is the five-dimensional tensor density with $\epsilon^{01234}=-1$, and $F_{\mu \nu}=\partial_{\mu} A_{\nu}-\partial_{\nu} A_{\mu}$ denotes the abelian field-strength tensor. The Einstein and gauge field equations of motion derived from Lagrangian (11) are

$$
\begin{aligned}
& R_{\mu \nu}-\frac{1}{2} g_{\mu \nu} R=2\left(F_{\mu \alpha} F_{\nu}^{\alpha}-\frac{1}{4} g_{\mu \nu} F_{\rho \sigma} F^{\rho \sigma}\right), \\
& \nabla_{\nu}\left(F^{\mu \nu}+\frac{1}{\sqrt{3} \sqrt{-g}} \epsilon^{\mu \nu \lambda \rho \sigma} A_{\lambda} F_{\rho \sigma}\right)=0
\end{aligned}
$$

Parameterized by four constants $(\mu, a, q, j)$, which correspond to the mass, the angular momentum, the electric charge and the scale of the Gödel background, respectively, the EMCS-Gödel black hole satisfying Eq. (2) takes the form [8]

$$
\begin{aligned}
d s^{2}= & -f(r)\left[d t+\frac{h(r)}{f(r)}(d \phi+\cos \theta d \psi)\right]^{2}+\frac{1}{4} r^{2}\left(d \theta^{2}+\sin ^{2} \theta d \psi^{2}\right) \\
& +\frac{d r^{2}}{V(r)}+\frac{r^{2} V(r)}{4 f(r)}(d \phi+\cos \theta d \psi)^{2}, \\
A= & B(r) d t+C(r)(d \phi+\cos \theta d \psi),
\end{aligned}
$$


where

$$
\begin{aligned}
f(r)= & 1-\frac{2 \mu}{r^{2}}+\frac{q^{2}}{r^{4}}, \\
h(r)= & j r^{2}+3 j q+\frac{(2 \mu-q) a}{2 r^{2}}-\frac{q^{2} a}{2 r^{4}}, \\
V(r)= & 1-\frac{2 \mu}{r^{2}}+\frac{8 j(\mu+q)[a+2 j(\mu+2 q)]}{r^{2}}+\frac{2(\mu-q) a^{2}}{r^{4}} \\
& +\frac{q^{2}\left[1-16 j a-8 j^{2}(\mu+3 q)\right]}{r^{4}}, \\
B(r)= & \frac{\sqrt{3} q}{2 r^{2}}, \quad C(r)=\frac{\sqrt{3}}{2}\left(j r^{2}+2 j q-\frac{q a}{2 r^{2}}\right) .
\end{aligned}
$$

In the above equations, the Euler angles $\theta, \psi$ and $\phi$ run over the ranges 0 to $\pi, 0$ to $2 \pi$ and 0 to $4 \pi$, respectively. The line element (3) is the charged generalization of the Kerr Gödel black hole. It is asymptotically rotating. Just as its uncharged counterpart, it exhibits the peculiar features such as the presence of closed time-like curves and the absence of globally spatial-like Cauchy surface. When the electric charge parameter $q=0$, it returns to the Kerr Gödel black hole in [4], whose Hawking radiation has been investigated via the covariant anomalies and effective action [21]. The angular velocities and the electro-static potential of the EMCS-Gödel black hole are given by

$$
\begin{aligned}
\Omega(r) & =\Omega_{\phi}=h(r) / U(r), \quad \Omega_{\psi}=0 \\
\Phi & =\ell^{\mu} A_{\mu}=B(r)+\Omega_{\phi} C(r)
\end{aligned}
$$

where

$$
\begin{aligned}
U(r) & =\frac{r^{2} V(r)-4 h^{2}(r)}{4 f(r)} \\
& =-j^{2} r^{2}\left(r^{2}+2 \mu+6 q\right)+3 j q a+\frac{(\mu-q) a^{2}}{2 r^{2}}-\frac{q^{2} a^{2}}{4 r^{4}}+\frac{r^{2}}{4},
\end{aligned}
$$

and the corotating vector $\ell=\partial_{t}+\Omega(r) \partial_{\phi}$. With help of this vector, the surface gravity $\kappa$ is defined by $\kappa^{2}=$ $-\left.\frac{1}{2} \ell_{\mu ; \nu} \ell^{\mu ; \nu}\right|_{r=r_{+}}$, where the outside event horizon $r_{+}$is determined by equation $V\left(r_{+}\right)=0$ and reads

$$
\begin{aligned}
r_{+}^{2}= & \mu-4 j(\mu+q) a-8 j^{2}(\mu+q)(\mu+2 q)+\sqrt{\delta}, \\
\delta= & {\left[\mu-q-8 j^{2}(\mu+q)^{2}\right] } \\
& \times\left[\mu+q-2 a^{2}-8 j(\mu+2 q) a-8 j^{2}(\mu+2 q)^{2}\right] .
\end{aligned}
$$

Hence Hawking temperature via the surface gravity formula is read off as

$$
T_{H}=\frac{\kappa}{2 \pi}=\frac{r_{+} V^{\prime}\left(r_{+}\right)}{8 \pi \sqrt{U\left(r_{+}\right)}} .
$$

Here, and in what follows, the prime' denotes the derivative with respect to the radial coordinate $r$. The entropies via the Bekenstein-Hawking area law are

$$
S=\pi^{2} r_{+}^{2} \sqrt{U\left(r_{+}\right)} .
$$

It is worth noting that the electro-static potential (7) is not zero at infinity, but $\Phi_{\infty}=-\sqrt{3} / 2$, since the Gödel universe possesses a global rotation at infinity. In order to make the electro-static potential vanish at infinity, we can rescale the gauge field (4) as

$$
A=\hat{B}(r) d t+C(r)(d \phi+\cos \theta d \psi)
$$

where $\hat{B}(r)=B(r)+\sqrt{3} / 2$. We shall adopt Eq. (12) for all the calculations related to gauge fields.

Now, we compute the mass, angular momenta, and electric charge of the EMCS-Gödel black hole. Because of the presence of closed time-like curves and the special asymptotical structure of the Gödel-type black hole, naive application of the traditional approaches, such as the methods of Komar integral, the usual Abbott-Deser construction and the covariant phase space [40], fails to give conserved charges in agreement with the first law of thermodynamics. 
In [9], a new method, based on cohomological techniques [15], has been successfully used to derive the conserved charges of the Kerr Gödel black hole. This method is also applicable to the EMCS-Gödel black hole. Our computation follows work [9]. Here we only give the formulas closely relevant to our calculations. For more details see [9, 15].

Let $\varphi^{i}=\left(g_{\mu \nu}, A_{\mu}\right)$ denote the fields of the five-dimensional ungauged minimal supergravity. $\bar{\varphi}^{i}=\left(\bar{g}_{\mu \nu}, \bar{A}_{\mu}\right)$ is any fixed reference solution of the motion equtions in Eq. (2). Consider the linearized theory for the variables $\delta \varphi^{i}=\varphi^{i}-\bar{\varphi}^{i}=\left(\delta g_{\mu \nu}, \delta A_{\mu}\right)=\left(h_{\mu \nu}, a_{\mu}\right)$. The equivalence classes of conserved 3-forms of this linearized theory are in correspondence with equivalence classes of field dependent gauge parameters $\xi^{\mu}(x)$ and $\Lambda(x)$ satisfying the reducibility equations [9]

$$
\begin{aligned}
& \mathcal{L}_{\xi} \bar{g}_{\mu \nu}=0, \\
& \mathcal{L}_{\xi} \bar{A}_{\mu}+\partial_{\mu} \Lambda=0 .
\end{aligned}
$$

Each pair of solutions $(\xi, \Lambda)$ of Eq. (13) is associated with a conserved 3 -form $k_{\xi, \Lambda}[\delta \varphi, \bar{\varphi}]$ that can be obtained by computing the weakly vanishing Noether currents related to the gauge transformations. Obviously, when $\xi$ is a Killing vector $\bar{\xi}$ of the background $\bar{\varphi}$ and $\Lambda$ is a constant $c$, Eq. (13) holds. For the solutions $(\bar{\xi}, 0)$, the conserved 3 -form $k_{\xi, \Lambda}$ can be decomposed as $k_{\bar{\xi}, 0}=k_{\bar{\xi}}^{g r}+k_{\bar{\xi}}^{e m}+k_{\bar{\xi}}^{C S}$, where $k_{\bar{\xi}}^{g r}, k_{\bar{\xi}}^{e m}$ and $k_{\bar{\xi}}^{C S}$ are the contributions from gravitation, electromagnetism and the Chern-Simons term, respectively. $k_{\bar{\xi}}^{g r}$ is defined by

$$
k_{\bar{\xi}}^{g r}[h, \bar{g}]=-\delta K_{\bar{\xi}}^{K}-\bar{\xi} \cdot \Theta^{g r},
$$

where the Komar 3-form

$$
\begin{aligned}
K_{\bar{\xi}}^{K} & =\frac{\sqrt{-g}}{192 \pi}\left(\nabla^{\mu} \bar{\xi}^{\nu}-\nabla^{\nu} \bar{\xi}^{\mu}\right) \epsilon_{\mu \nu \lambda \rho \sigma} d x^{\lambda} \wedge d x^{\rho} \wedge d x^{\sigma}, \\
\Theta^{g r} & =\frac{\sqrt{-\bar{g}}}{384 \pi}\left(\bar{\nabla}_{\alpha} h^{\mu \alpha}-\bar{\nabla}^{\mu} h\right) \epsilon_{\mu \nu \lambda \rho \sigma} d x^{\nu} \wedge d x^{\lambda} \wedge d x^{\rho} \wedge d x^{\sigma},
\end{aligned}
$$

and $\bar{\xi} \cdot=\bar{\xi}^{\mu} \frac{\partial}{\partial\left(d x^{\mu}\right)}$. The electromagnetic contribution $k_{\bar{\xi}}^{e m}$ is similar with $k_{\bar{\xi}}^{g r}$, which reads

$$
k_{\bar{\xi}}^{e m}[a, h ; \bar{A}, \bar{g}]=-\delta Q_{\bar{\xi}, 0}^{e m}-\bar{\xi} \cdot \Theta^{e m},
$$

where

$$
\begin{aligned}
& Q_{\bar{\xi}, c}^{e m}=\frac{\sqrt{-g}}{48 \pi}\left[\left(\bar{\xi}^{\alpha} A_{\alpha}+c\right) F^{\mu \nu}\right] \epsilon_{\mu \nu \lambda \rho \sigma} d x^{\lambda} \wedge d x^{\rho} \wedge d x^{\sigma}, \\
& \Theta^{e m}=\frac{\sqrt{-\bar{g}}}{96 \pi}\left(\bar{F}^{\alpha \mu} a_{\alpha}\right) \epsilon_{\mu \nu \lambda \rho \sigma} d x^{\nu} \wedge d x^{\lambda} \wedge d x^{\rho} \wedge d x^{\sigma} .
\end{aligned}
$$

The contribution from the Chern-Simons term is

$$
k_{\bar{\xi}}^{C S}[a, \bar{A}]=\frac{1}{2 \sqrt{3} \pi}\left(\bar{\xi}^{\alpha} \bar{A}_{\alpha}\right) a_{\rho} \bar{F}_{\mu \nu} d x^{\rho} \wedge d x^{\mu} \wedge d x^{\nu} .
$$

For the solution $(0,1)$, which corresponds to the contribution from the electric charge, the conserved 3-form

$$
k_{0,1}[a, h ; \bar{A}, \bar{g}]=-\delta\left(Q_{0,1}^{e m}+\mathbb{J}\right),
$$

where

$$
\mathbb{J}=-\frac{1}{4 \sqrt{3} \pi} A_{\rho} F_{\mu \nu} d x^{\rho} \wedge d x^{\mu} \wedge d x^{\nu} .
$$

Take into account a path $\gamma$ in the space of solutions that interpolates between a given solution $\varphi$ and the background solution $\bar{\varphi}$. Let $d_{V} \varphi$ be a one-form in the field space. As long as the pair $(\bar{\xi}, c)$ satisfy Eq. (13) for all solutions along this path, we can get a closed 3 -form

$$
K_{\bar{\xi}, c}=\int_{\gamma} k_{\bar{\xi}, c}\left[d_{V} \varphi ; \varphi\right]
$$

i.e. $d K_{\bar{\xi}, c}=0$ in a four-dimensional hypersurface $\Sigma$. Using Eq. (23), one can define conserved charges

$$
Q_{\bar{\xi}, c}=\oint_{S} K_{\bar{\xi}, c},
$$


where the three-dimensional closed surface $S$ is the boundary of the hypersurface $\Sigma$.

Next we turn our attention to calculate the conserved charges of the EMCS-Gödel black hole via (24). The mass is computed as

$$
\begin{aligned}
M & =\oint_{S} K_{\partial / \partial_{t}, 0} \\
& =\frac{3}{4} \pi(m+q)-4 \pi(m+q) j a-8 \pi(m+2 q)(m+q) j^{2} .
\end{aligned}
$$

The angular momentum along the $\phi$ direction

$$
\begin{aligned}
J_{\phi}= & -\oint_{S} K_{\partial / \partial_{\phi}, 0} \\
= & \frac{1}{2} \pi\left\{a\left[m-\frac{q}{2}-2(m-q) a j-8\left(m^{2}+m q-2 q^{2}\right) j^{2}\right]\right. \\
& \left.-3 j q^{2}+8(3 m+5 q) j^{2} q^{2}\right\},
\end{aligned}
$$

while the one with respect to the coordinate $\psi$ is zero. The electric charge is given by

$$
\begin{aligned}
Q & =\oint_{S} K_{0,1} \\
& =\frac{\sqrt{3}}{2} \pi\left[q-4(m+q) a j-8(m+q) q j^{2}\right] .
\end{aligned}
$$

The electric charge can also be computed through

$$
Q=\frac{1}{4 \pi} \int_{S_{3}}\left(\frac{1}{12} \sqrt{-g} F^{\alpha \beta} \epsilon_{\alpha \beta \rho \mu \nu}-\frac{1}{\sqrt{3}} A_{\rho} F_{\mu \nu}\right) d x^{\rho} \wedge d x^{\mu} \wedge d x^{\nu},
$$

where the integration is performed on the 3 -sphere at infinity. All the conserved charges are consistent with the first law of thermodynamics

$$
\begin{aligned}
d M & =T_{H} d S+\Omega_{+} d J_{\phi}+\Phi_{+} d Q+W d j, \\
\frac{2}{3} M & =T_{H} S+\Omega_{+} J_{\phi}+\frac{2}{3} \Phi_{+} Q-\frac{1}{3} W j,
\end{aligned}
$$

where $\Omega_{+}=\Omega\left(r_{+}\right)$and $\Phi_{+}=\hat{B}\left(r_{+}\right)+\Omega\left(r_{+}\right) C\left(r_{+}\right)$are the angular velocity and the electro-static potential at the event horizon, and

$$
W=2 \pi(m+q)[a+2 j(m+2 q)]
$$

is the generalized force conjugate to the Gödel parameter $j$ since we have considered $j$ as a thermodynamical variable to close the expression of the integral Bekenstein-Smarr formula.

\section{HAWKING FLUXES AND COVARIANT ANOMALIES}

In this section, we shall investigate Hawking radiation of the EMCS-Gödel black hole [8] via the covariant gravitational and gauge anomaly cancellation method [27] developed on basis of [16 18]. The same results will be obtained if we adopt the consistent anomaly cancellation method in [16 18]. Before our proceeding, it is necessary for us to briefly review this approach. By performing the technique of dimensional reduction, the massless scalar field near the horizon can be effectively described by a collection of scalar fields in the background of (1+1)-dimensional spacetime. Thereby we can treat the higher dimensional theory as a $(1+1)$-dimensional effective theory near the horizon. If we omit the classically irrelevant ingoing modes inside the horizon, the two dimensional effective theory becomes chiral. Such a chiral theory exhibits covariant gravitational and gauge anomalies. Imposing the boundary condition that the covariant energy momentum tensor and current vanish at the horizon, we can get fluxes that just cancel these anomalies and are identified with Hawking fluxes for the energy momentum tensor and charges. 
We first implement a process of dimensional reduction by considering the free part of the action for a scalar massless complex field in the background of metric (3) and gauge field (12). We have

$$
\begin{aligned}
S[\varphi]= & \frac{1}{2} \int d^{5} x \varphi^{*} \mathcal{D}_{\mu}\left(\sqrt{-g} g^{\mu \nu} \mathcal{D}_{\nu} \varphi\right) \\
= & \frac{1}{16} \int d t d r d \theta d \phi d \psi \sin \theta \varphi^{*}\left\{-\frac{4 r U(r)}{V(r)}\left(\mathcal{D}_{t}+\Omega(r) \mathcal{D}_{\phi}\right)^{2}+\partial_{r}\left[r^{3} V(r) \partial_{r}\right]\right. \\
& \left.\quad+\frac{r^{3}}{U(r)} \mathcal{D}_{\phi}^{2}+4 r\left[\frac{1}{\sin \theta} \partial_{\theta}\left(\sin \theta \partial_{\theta}\right)+\frac{\left(\partial_{\psi}-\cos \theta \partial_{\phi}\right)^{2}}{\sin ^{2} \theta}\right]\right\} \varphi
\end{aligned}
$$

where $\mathcal{D}_{\mu}=\partial_{\mu}+i e A_{\mu}$. After performing a partial wave decomposition $\varphi=\sum_{l m n} \varphi_{l m n}(t, r) \exp (i m \phi+i n \psi) \Theta_{l m n}(\theta)$, where the spin-weighted spheroidal functions $\Theta_{l m n}(\theta)$ satisfy

$$
\left[\frac{1}{\sin \theta} \partial_{\theta}\left(\sin \theta \partial_{\theta}\right)-\frac{(n-m \cos \theta)^{2}}{\sin ^{2} \theta}+l(l+1)-m^{2}\right] \Theta_{l m n}(\theta)=0,
$$

and only keeping the dominant terms near the horizon, the action (31) becomes

$$
\begin{gathered}
S[\varphi] \simeq \frac{1}{8} \sum_{l m n} \int d t d r r^{2} \sqrt{U(r)} \varphi_{l m n}^{*}\left\{-\frac{1}{F(r)}\left[\partial_{t}+i e(\hat{B}(r)+\Omega(r) C(r))\right.\right. \\
\left.+i m \Omega(r)]^{2}+\partial_{r}\left[F(r) \partial_{r}\right]\right\} \varphi_{l m n} .
\end{gathered}
$$

In Eq. (33), we have defined $2 F(r)=r V(r) U(r)^{-1 / 2}$. Thereby the physics near the horizon can be described by an infinite set of effective massless fields on a $(1+1)$-dimensional spacetime with the metric and the gauge potential

$$
\begin{aligned}
d s^{2} & =-F(r) d t^{2}+\frac{d r^{2}}{F(r)}, \\
\mathcal{A}_{t} & =e \mathcal{A}_{t}^{(0)}+m \mathcal{A}_{t}^{(1)}=e[\hat{B}(r)+\Omega(r) C(r)]+m \Omega(r), \quad \mathcal{A}_{r}=0,
\end{aligned}
$$

where $\mathcal{A}_{t}(\infty)=0$, and $F(\infty)=F^{\prime}(\infty)=F^{\prime \prime}(\infty)=0$. In such a two dimensional effective theory, the $t$-component of the gauge field $\mathcal{A}$ contains two types of U(1) fields. the gauge field $\mathcal{A}_{t}^{(0)}$ comes from the original electric field (12), while $\mathcal{A}_{t}^{(1)}$ can be interpreted as an induced $U(1)$ gauge field from the axial isometry in the $\phi$ direction. The azimuthal quantum number $m$ for each partial wave serves as charges of the gauge field $\mathcal{A}_{t}^{(1)}$.

Next, we pay our attention to derive the currents of the gauge field (35) via covariant gauge anomaly. In our case, there are two $U(1)$ gauge symmetries yielding two gauge currents $J^{(0) r}$ and $J^{(1) r}$, corresponding to the gauge potentials $\mathcal{A}_{t}^{(0)}$ and $\mathcal{A}_{t}^{(1)}$, respectively. Except for different types of charges, both the gauge potentials are essentially consistent with each other. Thus we only give an explicit derivation of the current $J^{(0) r} . J^{(1) r}$ can be obtained by a similar procedure.

Due to the anomaly cancellation method, the gauge current behaves differently in the range outside the horizon and that near the horizon. In the former, namely, the range $r \in\left[r_{+}+\varepsilon,+\infty\right)$, the current $J_{(O)}^{(0) \mu}$ is anomaly free and takes the conserved form

$$
\nabla_{\mu} J_{(O)}^{(0) \mu}=0
$$

while in the range near the horizon $\left(r \in\left[r_{+}, r_{+}+\varepsilon\right]\right)$, because of the breakdown of the classical gauge symmetry, the current $J_{(H)}^{(0) \mu}$ satisfies the anomaly Ward identity [17, 18, 27]

$$
\nabla_{\mu} \frac{1}{e} J_{(H)}^{(0) \mu}=\frac{-1}{4 \pi \sqrt{-g}} \epsilon^{\alpha \beta} \mathcal{F}_{\alpha \beta},
$$

where $\epsilon^{\alpha \beta}$ is an antisymmetry tensor density with $\epsilon^{t r}=-\epsilon_{t r}=1$ and $\mathcal{F}_{\alpha \beta}=\partial_{\alpha} \mathcal{A}_{\beta}-\partial_{\beta} \mathcal{A}_{\alpha}$. Solving Eqs. (36) and (37), we have

$$
\begin{aligned}
\sqrt{-g} J_{(O) r}^{(0) r} & =c_{O}^{(0)}, \\
\sqrt{-g} J_{(H)}^{(0) r} & =c_{H}^{(0)}+\frac{e}{2 \pi}\left[\mathcal{A}_{t}(r)-\mathcal{A}_{t}\left(r_{+}\right)\right],
\end{aligned}
$$


where the charge flux $c_{O}^{(0)}$ and $c_{H}^{(0)}$ are two integration constants, which denote the current at infinity and the one at the horizon, respectively. Introducing two step functions $\Theta(r)=\Theta\left(r-r_{+}-\varepsilon\right)$ and $H(r)=1-\Theta(r)$ to write the total current as

$$
J^{(0) \mu}=J_{(O)}^{(0) \mu} \Theta(r)+J_{(H)}^{(0) \mu} H(r)
$$

we find that the Ward identity becomes

$$
\partial_{r}\left[\sqrt{-g} J^{(0) r}\right]=\partial_{r}\left(\frac{e}{2 \pi} \mathcal{A}_{t} H\right)+\left\{\sqrt{-g}\left[J_{(O)}^{(0) r}-J_{(H)}^{(0) r}\right]+\frac{e}{2 \pi} \mathcal{A}_{t}\right\} \delta\left(r-r_{+}-\varepsilon\right) .
$$

In order to make the current preserve the gauge symmetry, the first term in the above equation must be cancelled by the classically irrelevant ingoing modes while the second term should vanish at the horizon, which yields

$$
c_{O}^{(0)}=c_{H}^{(0)}-\frac{e}{2 \pi} \mathcal{A}_{t}\left(r_{+}\right), \quad \mathcal{A}_{t}\left(r_{+}\right)=e\left[\hat{B}\left(r_{+}\right)+\Omega\left(r_{+}\right) C\left(r_{+}\right)\right]+m \Omega\left(r_{+}\right)
$$

Further imposing the boundary condition that the covariant current vanishes at the horizon, namely, $c_{H}^{(0)}=0$, then the charge flux corresponding to the gauge potential $\mathcal{A}_{t}^{(0)}$ is given by

$$
c_{O}^{(0)}=-\frac{e}{2 \pi} \mathcal{A}_{t}\left(r_{+}\right)
$$

Following the analysis of computing $c_{O}^{(0)}$ step by step, the current with respect to the gauge potential $\mathcal{A}_{t}^{(1)}$ reads

$$
c_{O}^{(1)}=-\frac{m}{2 \pi} \mathcal{A}_{t}\left(r_{+}\right)
$$

From Eq. (37), one can see that $J^{(0) r}$ and $J^{(1) r}$ are not independent for each oter but there exists the relation $\frac{1}{e} J^{(0) r}=\frac{1}{m} J^{(1) r}=\mathcal{J}^{r}$ between them, where $\mathcal{J}^{\mu}$ satisfies the covariant gauge anomaly equation

$$
\nabla_{\mu} \mathcal{J}_{(H)}^{\mu}=\frac{-1}{4 \pi \sqrt{-g}} \epsilon^{\alpha \beta} \mathcal{F}_{\alpha \beta},
$$

near the horizon. By analogy, the current out of the horizon can be solved as

$$
c_{O}=-\frac{1}{2 \pi} \mathcal{A}_{t}\left(r_{+}\right)
$$

With the expression of the charge flux in hand, we now consider the energy momentum flux in the way similar to the gauge anomaly. Near the horizon, if we eliminate the quantum effect of the ingoing modes, the invariance under general coordinate transformation will break down. Thus the two dimensional effective field theory will exhibit a gravitational anomaly. For the right-handed fields, the covariant gravitational anomaly has the form [27]

$$
\nabla_{\mu} T_{\nu}^{\mu}=\frac{1}{96 \pi \sqrt{-g}} \epsilon_{\nu \mu} \partial^{\mu} R=\frac{1}{\sqrt{-g}} \partial_{\mu} N_{\nu}^{\mu} .
$$

In the case of a background spacetime with the effective metric (34), the anomaly is timelike $\left(\nabla_{\mu} T_{t}^{\mu}=0\right)$, and

$$
N_{t}^{r}=\frac{1}{192 \pi}\left(2 F F^{\prime \prime}-F^{2}\right) .
$$

Because of the presence of the external gauge field $\mathcal{A}$, the energy momentum tensor outside the horizon does not take the conserved form but satisfies the Lorentz force law

$$
\nabla_{\mu} T_{(O) \nu}^{\mu}=\mathcal{F}_{\mu \nu} \mathcal{J}_{(O)}^{\mu}
$$

while the energy momentum near the horizon obeys the anomalous Ward identity after adding the gravitational anomaly,

$$
\nabla_{\mu} T_{(H) \nu}^{\mu}=\mathcal{F}_{\mu \nu} \mathcal{J}_{(H)}^{\mu}+\frac{1}{96 \pi \sqrt{-g}} \epsilon_{\nu \mu} \partial^{\mu} R
$$


Solving both the equations (48) and (49) for the $\nu=t$ component, we get

$$
\begin{aligned}
& \sqrt{-g} T_{(O) t}^{r}=a_{O}+c_{O} \mathcal{A}_{t}(r), \\
& \sqrt{-g} T_{(H) t}^{r}=a_{H}+\left.\left[c_{O} \mathcal{A}_{t}(r)+\frac{1}{4 \pi} \mathcal{A}_{t}^{2}(r)+N_{t}^{r}\right]\right|_{r_{+}} ^{r},
\end{aligned}
$$

where $a_{O}$ and $a_{H}$ are two constants, corresponding to the fluxes at infinity and horizon, respectively. Similar to the case of the gauge current, we express the total energy momentum tensor as a sum of two combinations $T_{\nu}^{\mu}=$ $T_{(O) \nu}^{\mu} \Theta(r)+T_{(H) \nu}^{\mu} H(r)$. Using Eqs. (50a) and (50b), we find

$$
\begin{aligned}
\sqrt{-g} \nabla_{\mu} T_{t}^{\mu}= & c_{O} \partial_{r} \mathcal{A}_{t}+\partial_{r}\left[\left(\frac{1}{4 \pi} \mathcal{A}_{t}^{2}+N_{t}^{r}\right) H\right] \\
& +\left[\sqrt{-g}\left(T_{(O) t}^{r}-T_{(H) t}^{r}\right)+\frac{1}{4 \pi} \mathcal{A}_{t}^{2}+N_{t}^{r}\right] \delta\left(r-r_{+}-\varepsilon\right)
\end{aligned}
$$

In the above equation, the first term is the classical effect of the background $\mathrm{U}(1)$ gauge field for constant current flow. The second term should be cancelled by the quantum effect of the classically irrelevant ingoing modes. In order to guarantee the energy momentum tensor is invariant under general coordinate transformations, the third term must vanish at the horizon, which yields

$$
a_{O}=a_{H}+\frac{1}{4 \pi} \mathcal{A}_{t}^{2}\left(r_{+}\right)+\frac{1}{192 \pi} F^{2}\left(r_{+}\right),
$$

where we have used $N_{t}^{r}\left(r_{+}\right)=-F^{2}\left(r_{+}\right) /(192 \pi)$. As what we have done to evaluate the gauge current at infinity, to fix $a_{O}$ completely, we require to impose the boundary condition that the covariant energy momentum tensor vanishes at horizon, i.e., $a_{H}=0$. We will see that such a boundary condition is compatible with the Unruh vacuum in the next section. Therefore, the total flow of energy momentum tensor is

$$
a_{O}=\frac{1}{4 \pi} \mathcal{A}_{t}^{2}\left(r_{+}\right)+\frac{\kappa^{2}}{48 \pi}, \quad \kappa=\frac{1}{2} F^{\prime}\left(r_{+}\right)=\frac{r_{+} V^{\prime}\left(r_{+}\right)}{4 \sqrt{U\left(r_{+}\right)}},
$$

For the sake of comparing the total energy momentum flux (53) with the Hawking one, we consider Hawking radiation with the Fermionic Plank distribution $N_{e, m}(\omega)=1 /\left(e^{\left[\omega-e \hat{\Phi}_{+}-m \Omega\left(r_{+}\right)\right] / T_{H}}+1\right)$ in the background of the EMCS-Gödel black hole, where $T_{H}$ is the Hawking temperature (10) via surface gravity formula, $\hat{\Phi}_{+}=\hat{B}\left(r_{+}\right)+\Omega\left(r_{+}\right) C\left(r_{+}\right)$is the electric chemical potential of the gauge field (12) at the horizon and $\Omega\left(r_{+}\right)$is the angular velocity at the horizon. The Hawking flux with this distribution is

$$
F_{M}=\int_{0}^{\infty} \frac{d \omega}{2 \pi} \omega\left[N_{e, m}(\omega)+N_{-e,-m}(\omega)\right]=\frac{1}{4 \pi} \mathcal{A}_{t}^{2}\left(r_{+}\right)+\frac{\kappa^{2}}{48 \pi}
$$

which takes the same form as Eq. (53). This implies that we have reproduced the Hawking temperature (10) via the covariant anomaly cancellation method.

\section{HAWKING FLUXES AND EFFECTIVE ACTION}

In this section, we will use the effective action method to exploit Hawking radiation of the EMCS-Gödel black hole in background of the two dimensional metric (34) and gauge field (35). In two dimensional effective theory, there exist normal effective action and chiral effective action. The former describes the effective theory away from the event horizon. The energy momentum tensor and gauge current induced from this action are anomaly free and take consistent forms. The normal effective action has been used to derive the Hawking fluxes of the ReissnerNordström black hole [18]. On the other hand, the chiral effective action [31, 32] depicts the chiral theory, in which the energy momentum tensor and gauge current are not conserved but covariantly anomalous. By adopting the covariant boundary condition at the event horizon, this effective action can be applied to compute the Hawking fluxes of black holes [31]. In our work [25], the chiral effective action method has been extended to reproduce the Hawking fluxes of the Schwarzschild black holes in the isotropic coordinates where the determinant of the metric vanishes at the horizon. 


\section{A. Normal effective action and Hawking fluxes}

In two dimensional effective theory, the normal effective action is obtained by functional integration of the conformal anomaly [18, 37]. It consists of the gravitational (Polyakov) part and the gauge part. From a variation of this effective action, we get the energy momentum tensor and gauge current [18, 31]

$$
\begin{aligned}
T_{\mu \nu}= & -\frac{1}{\pi}\left(\nabla_{\mu} \mathcal{B} \nabla_{\nu} \mathcal{B}-g_{\mu \nu} \frac{1}{2} \nabla^{\rho} \mathcal{B} \nabla_{\rho} \mathcal{B}\right) \\
& -\frac{1}{48 \pi}\left[\nabla_{\mu} \mathcal{G} \nabla_{\nu} \mathcal{G}-2 \nabla_{\mu} \nabla_{\nu} \mathcal{G}+g_{\mu \nu}\left(2 R-\frac{1}{2} \nabla^{\rho} \mathcal{G} \nabla_{\rho} \mathcal{G}\right)\right], \\
J^{\mu}= & \frac{1}{\pi \sqrt{-g}} \epsilon^{\mu \nu} \partial_{\nu} \mathcal{B},
\end{aligned}
$$

where $R=-F^{\prime \prime}(r)$ is the Ricci scalar of the metric (34), and the two auxiliary fields $\mathcal{B}$ and $\mathcal{G}$ satisfy

$$
\nabla^{\mu} \nabla_{\mu} \mathcal{B}=-\frac{\epsilon^{\mu \nu}}{2 \sqrt{-g}} \mathcal{F}_{\mu \nu}, \quad \nabla^{\mu} \nabla_{\mu} \mathcal{G}=R
$$

From Eqs. (55) and (56), we find that the gauge current takes the conserved form $\nabla_{\mu} J^{\mu}=0$ while the energy momentum tensor obeys the Lorentz force law (48) and the trace anomaly

$$
\nabla_{\mu} T_{\nu}^{\nu}=\mathcal{F}_{\mu \nu} J^{\mu}, \quad T_{\mu}^{\mu}=-\frac{R}{24 \pi} .
$$

In the background of metric (34) and gauge field (35), solving Eq. (57), we get

$$
\begin{array}{rlrl}
\partial_{t} \mathcal{G} & =a, & \partial_{r} \mathcal{G} & =\frac{b-2 K}{F(r)}, \quad K=\frac{1}{2} F^{\prime}(r), \\
\partial_{t} \mathcal{B} & =\alpha, \quad \partial_{r} \mathcal{B}=\frac{\beta+\mathcal{A}_{t}(r)}{F(r)},
\end{array}
$$

where parameters $a, b, \alpha, \beta$ are constants. They can be determined by proper boundary conditions. As in the previous section, we still choose the boundary conditions that are compatible with the Unruh vacuum. Such a choice requires us to express the energy momentum tensor and gauge current in the Eddington-Finkelstein coordinate system $\{u, v\}$, where $u=t-r_{*}, v=t+r_{*}$, and $d r_{*}=d r / F(r)$. We have

$$
\begin{aligned}
T_{u u} & =-\frac{1}{4 \pi}\left(\alpha-\beta-\mathcal{A}_{t}(r)\right)^{2}-\frac{1}{192 \pi}\left[(a-b)^{2}-4 K^{2}+4 F(r) K^{\prime}\right], \\
T_{u v} & =T_{v u}=-\frac{1}{96 \pi} F(r) F^{\prime \prime}(r), \\
T_{v v} & =-\frac{1}{4 \pi}\left(\alpha+\beta+\mathcal{A}_{t}(r)\right)^{2}-\frac{1}{192 \pi}\left[(a+b)^{2}-4 K^{2}+4 F(r) K^{\prime}\right], \\
J_{u} & =\frac{1}{2 \pi}\left(\alpha-\beta-\mathcal{A}_{t}(r)\right), \quad J_{v}=-\frac{1}{2 \pi}\left(\alpha+\beta+\mathcal{A}_{t}(r)\right) .
\end{aligned}
$$

Adopting the Unruh vacuum boundary conditions

$$
\begin{array}{lll}
J_{u}=0, & T_{u u}=0, & r=r_{+}, \\
J_{v}=0, & T_{v v}=0, & r \rightarrow+\infty,
\end{array}
$$

the constants $a, b, \alpha, \beta$ can be solved as

$$
a=-b= \pm \kappa, \quad \alpha=-\beta=\frac{1}{2} \mathcal{A}_{t}\left(r_{+}\right) .
$$

Substituting the four constants into the $(r, t)$-component of the energy momentum tensor and the $r$-component of the gauge current, which correspond to fluxes for Hawking radiation and the gauge field, respectively, we obtain

$$
T^{r}{ }_{t}=\frac{1}{4 \pi} \mathcal{A}_{t}\left(r_{+}\right)\left[\mathcal{A}_{t}\left(r_{+}\right)-2 \mathcal{A}_{t}(r)\right]+\frac{\kappa^{2}}{48 \pi}, \quad J^{r}=-\frac{1}{2 \pi} \mathcal{A}_{t}^{2}\left(r_{+}\right),
$$


where $J^{r}$ is a constant since the normal effective action describes the theory away from the horizon and the gauge current is conserved. Taking the limit at infinity, we derive the charge flow and the Hawking fluxes

$$
\begin{aligned}
J^{r}(r \rightarrow \infty) & =-\frac{1}{2 \pi} \mathcal{A}_{t}^{2}\left(r_{+}\right), \\
T_{t}^{r}(r \rightarrow \infty) & =\frac{1}{4 \pi} \mathcal{A}_{t}^{2}\left(r_{+}\right)+\frac{\kappa^{2}}{48 \pi},
\end{aligned}
$$

which agree with Eqs. (45) and (53) via the covariant anomalies in the previous section.

\section{B. Chiral effective action and Hawking fluxes}

Varying the chiral effective action, the covariant energy momentum tensor $\widetilde{T}_{\nu}^{\mu}$ and the covariant gauge current $\widetilde{J}^{\mu}$ read 31,32$]$

$$
\begin{aligned}
\widetilde{T}_{\mu \nu} & =-\frac{1}{4 \pi} D_{\mu} \mathcal{B} D_{\nu} \mathcal{B}-\frac{1}{96 \pi}\left(\frac{1}{2} D_{\mu} \mathcal{G} D_{\nu} \mathcal{G}-D_{\mu} D_{\nu} \mathcal{G}+g_{\mu \nu} R\right) \\
\widetilde{J}^{\mu} & =\frac{1}{2 \pi} D^{\mu} \mathcal{B}
\end{aligned}
$$

where the chiral covariant derivative $D_{\mu}=\sqrt{-g} \epsilon_{\mu \nu} D^{\nu}=\nabla_{\mu}+\sqrt{-g} \epsilon_{\mu \nu} \nabla^{\nu}, \widetilde{J}_{\mu}=\sqrt{-g} \epsilon_{\mu \nu} \widetilde{J}^{\nu}$, and the two auxiliary fields $\mathcal{B}$ and $\mathcal{G}$ have been defined by Eq. (57). In the chiral effective theory, the covariant energy momentum tensor and gauge current satisfy the anomalous Ward identities,

$$
\begin{aligned}
\nabla_{\mu} \widetilde{J}^{\mu} & =\frac{-1}{4 \pi \sqrt{-g}} \epsilon^{\rho \sigma} \mathcal{F}_{\rho \sigma}, \\
\nabla_{\mu} \widetilde{T}_{\nu}^{\mu} & =\mathcal{F}_{\mu \nu} \widetilde{J}^{\mu}+\frac{1}{96 \pi \sqrt{-g}} \epsilon_{\nu \mu} \partial^{\mu} R .
\end{aligned}
$$

The energy momentum tensor also obeys the covariant trace anomaly $\widetilde{T}_{\mu}^{\mu}=-R /(48 \pi)$. Operating the chiral covariant derivative on the auxiliary fields $\mathcal{G}$ and $\mathcal{B}$, we get

$$
\begin{aligned}
& D_{t} \mathcal{G}=-F(r) D_{r} \mathcal{G}=\tilde{a}-\tilde{b}+2 K, \\
& D_{t} \mathcal{B}=-F(r) D_{r} \mathcal{B}=\tilde{\alpha}-\tilde{\beta}-\mathcal{A}_{t}(r),
\end{aligned}
$$

where $\tilde{a}, \tilde{b}, \tilde{\alpha}$ and $\tilde{\beta}$ are constants. Their relations will be determined later. Now the $(r, t)$-component of the covariant energy momentum tensor and the covariant gauge current can be read off as

$$
\begin{aligned}
\widetilde{T}_{t}^{r} & =\frac{1}{4 \pi}\left(\tilde{\alpha}-\tilde{\beta}-\mathcal{A}_{t}(r)\right)^{2}+\frac{1}{192 \pi}\left[(\tilde{a}-\tilde{b})^{2}-4 K^{2}+4 F(r) K^{\prime}\right], \\
\widetilde{J}^{r} & =F(r) \widetilde{J}^{t}=-\frac{1}{2 \pi}\left(\tilde{\alpha}-\tilde{\beta}-\mathcal{A}_{t}(r)\right) .
\end{aligned}
$$

Here we do not present the other components of the energy momentum tensor $\widetilde{T}_{\nu}^{\mu}$, which are useless for computation of the Hawking flux. Finally, to derive the Hawking fluxes and currents for gauge fields, we need to impose the covariant boundary conditions that the covariant energy momentum tensor and gauge current vanish at the horizon [31], namely,

$$
\tilde{\alpha}=\tilde{\beta}+\mathcal{A}_{t}\left(r_{+}\right), \quad \tilde{a}=\tilde{b} \pm 2 \kappa
$$

Therefore, taking the asymptotic limit, we obtain the gauge currents and the fluxes for energy momentum tensor

$$
\begin{aligned}
\widetilde{J}^{r}(r \rightarrow \infty) & =-\frac{1}{2 \pi} \mathcal{A}_{t}^{2}\left(r_{+}\right) \\
\widetilde{T}_{t}^{r}(r \rightarrow \infty) & =\frac{1}{4 \pi} \mathcal{A}_{t}^{2}\left(r_{+}\right)+\frac{\kappa^{2}}{48 \pi} .
\end{aligned}
$$

They are in agreement with Eqs. (45) and (53). 
It is worth noting that the covariant boundary conditions adopted in this subsection and the previous section are compatible with the Unruh vacuum. To see this, as in the case of the normal effective action, we could express the energy momentum tensor and gauge current in the Eddington-Finkelstein coordinate system $\{u, v\}$. By changing $(\alpha, \beta, a, b)$ to $(\tilde{\alpha}, \tilde{\beta}, \tilde{a}, \tilde{b})$, respectively, we find $\widetilde{T}_{u u}=T_{u u}, \widetilde{T}_{u v}=T_{u v} / 2, \widetilde{T}_{v v}=0, \widetilde{J}_{u}=J_{u}$, and $\widetilde{J}_{v}=0$, where $\widetilde{T}_{v v}$ and $\widetilde{J}_{v}$ are zeros, since the two dimensional effective theory is chiral and there are no ingoing modes. Clearly, Eqs. (65a) and (65b) hold when one adopts the covariant boundary conditions (78).

\section{SUMMARY}

We have obtained the EMCS-Gödel black hole's [8] conserved charges, such as the mass, the angular momenta and the electric charge along the lines of $[9]$. They are consistent with the differential first law and the generalized integral Smarr formula of black hole thermodynamics provided that the Gödel parameter $j$ is a thermodynamical variable. The EMCS-Gödel black hole is an exact charged rotating solution in the five dimensional minimal supergravity with Gödel background. It has closed time-like curves through every point and the asymptotic structure with a global rotation. These peculiar properties lead to the failure of the traditional methods on calculating the conserved charges. A viable method was presented in [9]. Whether there exist other methods to compute the conserved charges of the EMCS-Gödel black hole is still open.

Besides, we have derived the Hawking fluxes of the EMCS-Gödel black hole via covariant gravitational and gauge anomalies, as well as the effective action. By applying the technique of dimensional reduction to the metric (3) and the gauge field (12), which has the vanishing electro-static potential at infinity, higher dimensional theory near the horizon can be effectively described by a two dimensional theory in the background of metric (34) and gauge field (35). The reduced gauge field consists of two U(1) fields, one from the original gauge field and the other from the axial isometry along the $\phi$ direction. On the basis of both the two dimensional metric and gauge field, by adopting the covariant boundary conditions that are compatible with the Unruh vacuum, the covariant anomaly cancellation method and the approach of the effective action, including the normal and chiral effective action, were used to derive the same Hawking fluxes as those from Planck distribution for blackbody radiation in the background of the EMCS-Gödel black hole.

Our results show that Hawking radiation is a quantum phenomenon taking place at the event horizon, since both the methods of the anomaly cancellation and the chiral effective action only rely on the quantum anomalies and boundary conditions at the event horizon. Besides, our calculation supports the anomaly cancellation method is applicable to the black holes in the five dimensional minimal supergravity with Gödel background. In some sense, the approach of the anomaly cancellation is universal except for the procedure of dimensional reduction for different background spacetime considered in each case. A further development of this work is to derive the entropy of the EMCS-Gödel black hole in the same two dimensional effective theory, like in [34, 38]. Our analysis in the present paper can be directly generalized to the squashed charged rotating black hole in the five dimensional Gödel universe [39].

Acknowledgments: S.Q. Wu thanks Dr. G. Compère for helpful discussions, particularly for sharing his program to calculate conserved charges. This work was partially supported by the Natural Science Foundation of China under Grant Nos. 10975058 and 10675051. J.J. Peng was also supported in part by a Graduate Innovation Foundation of HuaZhong Normal University.

[1] K. Gödel, Rev. Mod. Phys. 21, 447 (1949).

[2] J.P. Gauntlett, J.B. Gutowski, C.M. Hull, S. Pakis, and H.S. Reall, Class. Quantum Grav. 20, 4587 (2003), hep-th/0209114

[3] E.K. Boyda, S. Ganguli, P. Horava, and U. Varadarajan, Phys. Rev. D 67, 106003 (2003), hep-th/0212087; T. Harmark and T. Takayanagi, Nucl. Phys. B 662, 3 (2003), hep-th/0301206

[4] E.G. Gimon and A. Hashimoto, Phys. Rev. Lett. 91, 021601 (2003), hep-th/0304181.

[5] C.A.R. Herdeiro, Nucl. Phys. B 665, 189 (2003), hep-th/0212002

[6] C.A.R. Herdeiro, Class. Quantum Grav. 20, 4891 (2003), hep-th/0307194.

[7] D. Brecher, U.H. Danielsson, J.P. Gregory, and M.E. Olsson, J. High Energy Phys. 11, 033 (2003), hep-th/0309058.

[8] S.Q. Wu, Phys. Rev. Lett. 100, 121301 (2008), arXiv:0709.1749 [hep-th].

[9] G. Barnich and G. Compère, Phys. Rev. Lett. 95, 031302 (2005), hep-th/0501102

[10] C. Stelea, K. Schleich, and D. Witt, Phys. Rev. D 78, 124006 (2008), arXiv:0807.4338 [hep-th].

[11] D. Klemm and L. Vanzo, Fortsch. Phys. 53, 919 (2005), hep-th/0411234.

[12] R. Kerner and R.B. Mann, Phys. Rev. D 75, 084022 (2007), hep-th/0701107; S. Bhattacharya and A. Saha, arXiv:0904.3441 $[\mathrm{gr}-\mathrm{qc}]$. 
[13] R.A. Konoplya and E. Abdalla, Phys. Rev. D 71, 084015 (2005), hep-th/0503029 S.B. Chen, B. Wang, and J.L. Jing, Phys. Rev. D 78, 064030 (2008), arXiv:0806.2177 [gr-qc]; W.B. Li, L.X. Xu, and M.L. Liu, Class. Quantum Grav. 26, 055008 (2009).

[14] J.J. Peng and S.Q. Wu, Phys. Lett. B 673, 216 (2009), arXiv:0901.0311 [hep-th].

[15] G. Barnich and F. Brandt, Nucl. Phys. B 633, 3 (2002), hep-th/0111246

[16] S.P. Robinson and F. Wilczek, Phys. Rev. Lett. 95, 011303 (2005), gr-qc/0502074.

[17] S. Iso, H. Umetsu, and F. Wilczek, Phys. Rev. Lett. 96, 151302 (2006), hep-th/0602146

[18] S. Iso, H. Umetsu, and Frank Wilczek, Phys. Rev. D 74, 044017 (2006), hep-th/0606018

[19] K. Murata and J. Soda, Phys. Rev. D 74, 044018 (2006), hep-th/0606069.

[20] K. Umetsu, arXiv:1003.5534 [hep-th].

[21] Q.Q. Jiang and X. Cai, Phys. Lett. B 677, 179 (2009).

[22] A.P. Porfyriadis, Phys. Rev. D 79, 084039 (2009), arXiv:0811.2822 [hep-th]; Z.B. Xu and B. Chen, Phys. Rev. D 75, 024041 (2007), hep-th/0612261; S. Iso, T. Morita, and H. Umetsu, JHEP 04, 068 (2007), hep-th/0612286 H. Shin and W. Kim, J. High Energy Phys. 06, 012 (2007), arXiv:0705.0265 [hep-th]; W. Kim and H. Shin, J. High Energy Phys. 07, 070 (2007), arXiv:0706.3563 [hep-th].

[23] L. Bonora and M. Cvitan, J. High Energy Phys. 05, 071 (2008), arXiv:0804.0198 [hep-th]; L. Bonora, M. Cvitan, S. Pallua, and I. Smolić, J. High Energy Phys. 12, 021 (2008), arXiv:0808.2360 [hep-th].

[24] E. Papantonopoulos and P. Skamagoulis, Phys. Rev. D 79, 084022 (2009), arXiv:0812.1759 [hep-th]; S.W. Wei, R. Li, Y.X. Liu, and J.R. Ren, Eur. Phys. J. C 65, 281 (2010), arXiv:0901.2614 [hep-th].

[25] S.Q. Wu, J.J. Peng, and Z.Y. Zhao, Class. Quantum Grav. 25, 135001 (2008), arXiv:0803.1338 [hep-th].

[26] U. Miyamoto and K. Murata, Phys. Rev. D 77, 024020 (2008), arXiv:0705.3150 [hep-th]; B. Chen and W. He, Class. Quantum Grav. 25, 135011 (2008), arXiv:0705.2984 [gr-qc].

[27] R. Banerjee and S. Kulkarni, Phys. Rev. D 77, 024018 (2008), arXiv:0707.2449 [hep-th]; R. Banerjee, Int. J. Mod. Phys. D 17, 2539 (2009), arXiv:0807.4637 [hep-th].

[28] R. Banerjee and B.R. Majhi, Phys. Rev. D 79, 064024 (2009), arXiv:0812.0497 [hep-th].

[29] J.J. Peng and S.Q. Wu, Phys. Lett. B 661, 300 (2008), arXiv:0801.0185 [hep-th].

[30] S. Nam and J.D. Park, Class. Quantum Grav. 26, 145015 (2009), arXiv:0902.0982 [hep-th]; S. Gangopadhyay and S. Kulkarni, Phys. Rev. D 77, 024038 (2008), arXiv:0710.0974 [hep-th].

[31] R. Banerjee and S. Kulkarni, Phys. Lett. B 659, 827 (2008), arXiv:0709.3916 [hep-th]; R. Banerjee and S. Kulkarni, Phys. Rev. D 79, 084035 (2009), arXiv:0810.5683 [hep-th].

[32] H. Leutwyler, Phys. Lett. B 153, 65 (1985); 155, 469(E) (1985).

[33] S. Gangopadhyay, Phys. Rev. D 77, 064027 (2008), arXiv:0712.3095 [hep-th]; S. Kulkarni, Class. Quantum Grav. 25, 225023 (2008), arXiv:0802.2456 [hep-th].

[34] L. Rodriguez and T. Yildirim, Class. Quantum Grav. 27, 155003 (2010), arXiv:1003.0026 [hep-th]; B.K. Button, L. Rodriguez, and C.A. Whiting, arXiv:1009.1661 [hep-th].

[35] A. Shirasaka and T. Hirata, arXiv:0804.1910 [hep-th].

[36] V. Mukhanov, A. Wipf, and A. Zelnikov, Phys. Lett. B 332 (1994) 283, hep-th/9403018; V.P. Frolov, W. Israel, and S.N. Solodukhin, Phys. Rev. D 54, 2732 (1996), hep-th/9602105 M. Buric, V. Radovanovic, and A. Mikovic, Phys. Rev. D 59, 084002 (1999), gr-qc/9804083 F.C. Lombardo, F.D. Mazzitelli, and J.G. Russo, Phys. Rev. D 59, 064007 (1999), gr-qc/9808048, S. Nojiri and S.D. Odintsov, Phys. Rev. D 57, 2363 (1998), hep-th/9706143 W. Kummer and D.V. Vassilevich, Ann. Phys. (Leipzig) 8, 801 (1999), gr-qc/9907041

[37] R. Balbinot and A Fabbri, Class. Quantum Grav. 20, 5439 (2003); R. Balbinot and A. Fabbri, Phys. Rev. D 59, 044031 (1999), hep-th/9807123 R. Balbinot and A. Fabbri, Phys. Lett. B 459, 112 (1999), gr-qc/9904034. R. Balbinot, A. Fabbri, and I. Shapiro, Nucl. Phys. B 559, 301 (1999), hep-th/9904162

[38] R. Banerjee, S. Gangopadhyay, and S. Kulkarni, Gen. Relativ. Gravit. 42, 2865 (2010), arXiv:0804.3492 [hep-th].

[39] S. Tomizawa, H. Ishihara, K. Matsuno, and T. Nakagawa, Prog. Theor. Phys. 121, 823 (2009), arXiv:0803.3873 [hep-th]

[40] V. Iyer and Robert M. Wald, Phys. Rev. D 50, 846 (1994); R.M. Wald and A. Zoupas, Phys. Rev. D 61, 084027 (2000), gr-qc/9911095. 